\title{
Coherent Reflection of Acoustic Plane Wave from Rough Interfaces in a Stratified Random Fluid Medium
}

Jin-Yuan Liu

Associate Professor, Institute of Undersea Technology, National Sun Yatsen University.

Shiahn-Wern Shyue

Associate Professor, Department of Marine Environment and Engineering, National Sun Yat-sen University.

Chen-Fen Huang

Ph.D. program, Scripps Institution of Oceanography in University of California at San Diego (Formerly, a graduate student in Institute of Undersea Technology).

Follow this and additional works at: https://jmstt.ntou.edu.tw/journal

Part of the Engineering Commons

\section{Recommended Citation}

Liu, Jin-Yuan; Shyue, Shiahn-Wern; and Huang, Chen-Fen (2000) "Coherent Reflection of Acoustic Plane Wave from Rough Interfaces in a Stratified Random Fluid Medium," Journal of Marine Science and Technology. Vol. 8: Iss. 2, Article 4.

DOI: $10.51400 / 2709-6998.2458$

Available at: https://jmstt.ntou.edu.tw/journal/vol8/iss2/4

This Research Article is brought to you for free and open access by Journal of Marine Science and Technology. It has been accepted for inclusion in Journal of Marine Science and Technology by an authorized editor of Journal of Marine Science and Technology. 
Coherent Reflection of Acoustic Plane Wave from Rough Interfaces in a Stratified Random Fluid Medium

Acknowledgements

This work was supported in part by National Science Council of Taiwan, R.O.C. through contracts NSC89-2611-M-110-012 and NSC89-2611-E-110-021. The authors would like to express our profound thanks for their financial supports. 


\title{
COHERENT REFLECTION OF ACOUSTIC PLANE WAVE FROM ROUGH INTERFACES IN A STRATIFIED RANDOM FLUID MEDIUM
}

\author{
Jin-Yuan Liu,* Shiahn-Wern Shyue** and Chen-Fen Huang***
}

Keywords: Acoustic Wave, Coherent Reflection, Rough Surface Scattering, Wave in Random Media, Horizontally-Stratified Media.

\section{ABSTRACT}

Coherent reflection of an acoustic plane wave from rough interfaces in a horizontally-stratified medium, within which the sound-speed distribution subject to a small and random perturbation, is considered in this paper. The general formulation based upon the theory for rough-interface scattering in a stratified medium, in conjunction with that for random medium is derived. The Green's function for a random medium bounded by two surfaces, which serves as generic solution of the problem, is obtained. The formulation is then applied to a simplified seabed environment to study the effects of surface roughness and medium inhomogenieties on the coherent reflection. The numerical results demonstrate that both the rough-surface scattering and medium scattering distract the energy from the coherent field, making the coefficient of the coherent reflection reduced with an amount, depending upon the degree of roughness and randomness measured by the relevant parameters. Furthermore, the characteristics of the distribution for the reflection coefficient with respect to incident angle show distinct feature, potentially allowing identification of dominant scattering mechanism.

\section{INTRODUCTION}

This paper considers coherent reflection of an acoustic plane wave impinging upon rough interfaces in

Paper Received Oct 26, 2000. Author for Correspondence: J. Y. Liu.

*Associate Professor, Institute of Undersea Technology, National Sun Yatsen University.

**Associate Professor, Department of Marine Environment and Engineering, National Sun Yat-sen University.

***Ph.D. program, Scripps Institution of Oceanography in University of California at San Diego (Formerly, a graduate student in Institute of Undersea Technology).

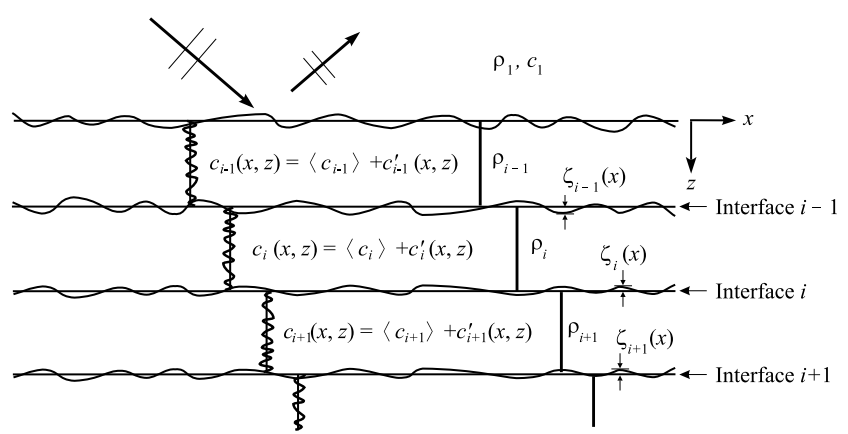

Fig. 1. Environmental model: a plane wave incident upon rough interfaces in a horizontally-stratified medium, within which the sound-speed distribution is subject to a small and random perturbation.

a horizontally-stratified medium, within which the sound speed is subject to small and random perturbation; the schematic diagram of the physical model is shown in Figure 1. This is an important and fundamental problem, in that the surface roughness and the medium inhomogenieties represent two basic types of perturbation interfering wave propagation in a bounded region. Furthermore, in many realistic applications, particularly for wave propagation in an oceanic waveguide, the surfaces bounding the media are rough, and the media supporting the waves are in general randomly inhomogeneous. As a result, two important aspects of the wave fields are affected, i.e., the transmission loss and the coherency of the sound field. For a wave propagating in such an environment, its energy may be attenuated due to scattering incurred by the rough boundaries as well as the random inhomogenieties inside the media, resulting in a severe loss of energy in addition to that due to traditional mechanisms such as geometric spreading and volumetric absorption. Moreover, the spatial distribution of the sound field no longer remains wellcorrelated, making the processing of acoustic signal a more difficult problem.

The surface roughness under consideration is due to boundary irregularities, while the medium inhomogenieties may originate from various sources, 
including random distributions of sound speed and/or density resulted from inhomogeneous constituents or dynamical perturbation of different origins, such as oceanographic mixing and turbulence. In the present analysis, attention is given only to the random soundspeed variations, in view of the fact that the distribution of the sound speed is often a greater concern than that of density for acoustic wave propagation in an oceanic environment. Evidently, the present problem involves two types of analysis, i.e., acoustic wave propagation in random media and wave interaction with rough surfaces; these two problems are traditionally treated separately. It is the main theme of this paper to consider the combined effects on acoustic reflection.

The analysis shall be based upon the theories for wave propagation in a horizontally-stratified medium, in combination with those for rough-surface and random-medium scattering; all of these disciplines are very broad subjects, and have attracted a great deal of interest for the researchers in the area. Related references on the above-mentioned subjects are so abundant that we place no intent to give an extensive survey, except to name a few that are most relevant to the present analysis. With respect to the theory on scattering from rough interfaces embedded in a horizontally-stratified media, a unified formulation developed by Kuperman and Schmidt $[16,17,18]$ has been proved to be accurate and efficient, so that it is adopted here. As for wave scattering due to medium inhomogenieties, a working formula for the mean-field solution for wave in a random slab similar to that due to Tang and Frisk [10, 27] for wave in a semi-infinite space shall be developed in this analysis. A few excellent books, such as those by Ewing, et al. [9], Brekhovskikh [4], and Jensen, at al. [15] for waves in stratified media, and those by Chernove [5], Ishimaru [13], Uscinski [28] for waves in random media, offer a comprehensive treatment on the subjects, and therefore serve as general references for this study.

In the following sections, we shall first present the general formulations, including the Green's functions and mean-field solution in the random media, and the coherent reflection from rough interfaces in a stratified medium, then the formulation is applied to a canonical problem to demonstrate the fundamental mechanisms. Many numerical results for the coherent reflection coefficient are generated and analyzed.

\section{FORMULATIONS}

Consider a monotonic acoustic plane wave with time dependence $e^{i w t}$, impinging upon rough surfaces on and/or embedded in a horizontally-stratified fluid medium, within which the sound-speed distribution is subject to a small and random perturbation as shown in
Figure 1. The sound speed in layer $i$ is:

$$
c_{i}=\left\langle c_{i}\right\rangle+c_{i}^{\prime}(\mathbf{r}, z)
$$

where $\left\langle c_{i}\right\rangle$ is the ensemble average over the layer $i$, and $c_{i}^{\prime}(\mathbf{r}, z)$ is the random variation of the sound speed, which has zero mean and is assumed to be small compared with $\left\langle c_{i}\right\rangle$; the density in layer $i$ is taken to be a constant value $\rho_{i}$. Furthermore, each layer $i$ is bounded by two rough surfaces (except for the upper and lower most semiinfinite media) at $z_{i-1}$ and $z_{i}$ with random elevations, respectively, equal to $z=\zeta_{i-1}(\mathbf{r})$ and $z=\zeta_{i}(\mathbf{r})$, which are also assumed to have zero means, and their magnitudes and slopes are small compared with the acoustic wavelength.

Under the above assumptions, the Helmholtz equation and the boundary conditions for the generic problem represented by layer $i$ may be shown to satisfy the following equations:

$$
\begin{aligned}
& \nabla^{2} p_{i}+\left\langle k_{i}\right\rangle^{2}\left(1-\frac{2 c_{i}^{\prime}}{\left\langle c_{i}\right\rangle}\right) p_{i}=0 \\
& p_{i-1}\left(\mathbf{r}, \zeta_{i-1}\right)=p_{i}\left(\mathbf{r}, \zeta_{i-1}\right) \\
& w_{i-1}\left(\mathbf{r}, \zeta_{i-1}\right)=w_{i}\left(\mathbf{r}, \zeta_{i-1}\right) \\
& p_{i}\left(\mathbf{r}, \zeta_{i}\right)=p_{i+1}\left(\mathbf{r}, \zeta_{i}\right) \\
& w_{i}\left(\mathbf{r}, \zeta_{i}\right)=w_{i+1}\left(\mathbf{r}, \zeta_{i}\right)
\end{aligned}
$$

where $p_{i}$ 's and $w_{i}$ 's are respectively the pressure and vertical displacement in the $i$-th medium, and the term involving wavenumber in Equation (2) is obtained by invoking the following approximation:

$$
\begin{aligned}
k_{i}^{2} & =\left(\frac{\omega}{\left\langle c_{i}\right\rangle+c_{i}^{\prime}}\right)^{2} \simeq\left[\frac{\omega}{\left\langle c_{i}\right\rangle}\left(1-\frac{c_{i}^{\prime}}{\left\langle c_{i}\right\rangle}\right)\right]^{2} \\
& \simeq\left\langle k_{i}\right\rangle^{2}\left(1-\frac{2 c_{i}^{\prime}}{\left\langle c_{i}\right\rangle}\right)
\end{aligned}
$$

It should be noted that $c_{i}^{\prime}$ in Equation (2) and $\zeta_{i-1}$, $\zeta_{i}$ in Equations (3) - (5) are stochastic variables, and in the most general case, they may be correlated. However, in this study, we shall assume that each randomness originates from different physical processes, and therefore they are assumed to be statistically independent.

The complete analysis for the acoustic fields in various layers is intrinsically complicated, in that the scattered field in each layer is a result of full reverberation in the waveguide. Although formally possible in terms of theoretical formulation, the solution of the 
problem becomes intractable even with a most powerful computational facility, because it involves a spectrum of waveguide incident components, each of which is scattered into all spectral components according to waveguide propagation. However, in the present study, we are primarily concerned with reflection of the coherent part of the sound fields. Under this consideration, only the first-order mean fields (to be defined below) are involved in the analysis. Therefore, the solution approach is first to obtain the mean-field solution in the various layers, then followed by the analysis of meanfield reflection from rough surfaces; that is the procedure to be developed below.

\section{Green's function and mean-field solutions in random media}

Here, we first derive the solution for wave propagation in a random slab, which constitutes the generic structure of this analysis. Following the common practice for wave propagation in a random medium, the solution for Equation (2) may be decomposed into a coherent mean field $\left\langle p_{i}\right\rangle$, and an incoherent scattered field $p_{i}^{s}$ :

$$
p_{i}=\left\langle p_{i}\right\rangle+p_{i}^{s}
$$

where it is assumed that $\left|p_{i}^{s}\right| \ll\left|\left\langle p_{i}\right\rangle\right|$. Substituting Equation (8) into Equation (2), and then taking the ensemble average results in the wave equation for the mean field:

$$
\nabla^{2}\left\langle p_{i}\right\rangle+\left\langle k_{i}\right\rangle^{2}\left\langle p_{i}\right\rangle=-\left\langle k_{i}\right\rangle^{2}\left\langle n_{i} p_{i}^{s}\right\rangle
$$

Furthermore, subtracting Equation (9) from Equation(2), then dropping the higher-order terms, yields the wave equation for the scattered field:

$$
\nabla^{2} p_{i}^{s}+\left\langle k_{i}\right\rangle^{2} p_{i}^{s}=-n_{i}\left\langle k_{i}\right\rangle^{2}\left\langle p_{i}\right\rangle
$$

where $n_{i}=-2 c_{i}^{\prime} /\left\langle c_{i}\right\rangle$ represents the randomness of the sound-speed variations. It is noted that the term on the right-hand-side of Equation (9), though high order in magnitude, must be retained so that the system is able to maintain consistency with the fact that the energy extracted from the coherent field by scattering may be appropriately accounted for.

Applying the Green's formula on Equation (10), and then substituting the resulted formulation into Equation (9) yields the equation for mean field and scattered field, respectively, given by

$$
\nabla^{2}\left\langle p_{i}\right\rangle+\left\langle k_{i}\right\rangle^{2}\left\langle p_{i}\right\rangle=-\frac{\left\langle k_{i}\right\rangle^{4}}{4 \pi} \int_{V\left(\mathbf{R}^{\prime}\right)}\left\langle n_{i}\left(\mathbf{R}^{\prime}\right) n_{i}(\mathbf{R})\right\rangle
$$

$$
\begin{aligned}
& \left\langle p_{i}\left(\mathbf{R}^{\prime}\right)\right\rangle G_{i}\left(\mathbf{R} ; \mathbf{R}^{\prime}\right) d V\left(\mathbf{R}^{\prime}\right) \\
& p_{i}^{s}(\mathbf{R})=\frac{\left\langle k_{i}\right\rangle^{2}}{4 \pi} \int_{V\left(\mathbf{R}^{\prime}\right)} n_{i}\left(\mathbf{R}^{\prime}\right)\left\langle p_{i}\left(\mathbf{R}^{\prime}\right)\right\rangle G_{i}\left(\mathbf{R} ; \mathbf{R}^{\prime}\right) d V\left(\mathbf{R}^{\prime}\right)
\end{aligned}
$$

where $\mathbf{R}=(\mathbf{r}, z)$, and $G_{i}\left(\mathbf{R} ; \mathbf{R}^{\prime}\right)$ is the Green's function in the random slab. It is seen that the mean field is governed by an integro-differential equation, and scattered field, which is excited by the mean field, may be derived, once the mean field is obtained. In the present study, we are primarily concerned with the coherent field so that only the solution for Equation (11) is in order. Equation (11) shows that, in order to obtain the solution for the mean field, it is necessary to derive the Green's function in the slab, which in present case requires to solve the following system of equations:

$$
\begin{aligned}
& \left(\nabla^{2}+k_{i-1}^{2}\right) G_{i-1}\left(\mathbf{R} ; \mathbf{R}^{\prime}\right)=0, z<0 \\
& \left(\nabla^{2}+\left\langle k_{i}\right\rangle^{2}\right) G_{i}\left(\mathbf{R} ; \mathbf{R}^{\prime}\right)=-4 \pi \delta\left(\mathbf{R}-\mathbf{R}^{\prime}\right), 0<z<d \\
& \left(\nabla^{2}+k_{i-1}^{2}\right) G_{i+1}\left(\mathbf{R} ; \mathbf{R}^{\prime}\right)=0, z>d
\end{aligned}
$$

subject to the following boundary conditions:

$$
\begin{aligned}
& \left.G_{i-1}\left(\mathbf{R} ; \mathbf{R}^{\prime}\right)\right|_{z=0}=\left.G_{i}\left(\mathbf{R} ; \mathbf{R}^{\prime}\right)\right|_{z=0} \\
& \left.\frac{1}{\rho_{i-1}} G_{i}\left(\mathbf{R} ; \mathbf{R}^{\prime}\right)\right|_{z=0}=\left.\frac{1}{\rho_{i}} \frac{\partial}{\partial z} G_{i}\left(\mathbf{R} ; \mathbf{R}^{\prime}\right)\right|_{z=0} \\
& \left.G_{i}\left(\mathbf{R} ; \mathbf{R}^{\prime}\right)\right|_{z=d}=\left.G_{i+1}\left(\mathbf{R} ; \mathbf{R}^{\prime}\right)\right|_{z=d} \\
& \left.\frac{1}{\rho_{i}} \frac{\partial}{\partial z} G_{i}\left(\mathbf{R} ; \mathbf{R}^{\prime}\right)\right|_{z=d}=\left.\frac{1}{\rho_{i+1}} \frac{\partial}{\partial z} G_{i+1}\left(\mathbf{R} ; \mathbf{R}^{\prime}\right)\right|_{z=d}
\end{aligned}
$$

Without loss of generality, the solution in each layer may be expressed by generalized Fourier integral:

$$
\begin{aligned}
G_{i-1}\left(\mathbf{R} ; \mathbf{R}^{\prime}\right) & =\int_{0}^{\infty} A e^{i k_{z, i-1} z} J_{0}\left(k_{r} r\right) k_{r} d k_{r} \\
G_{i}\left(\mathbf{R} ; \mathbf{R}^{\prime}\right) & =\int_{0}^{\infty} \frac{i}{k_{z, i}}\left(B e^{k_{z, o^{z}}}+C e^{-i k_{z, i} z}\right. \\
& \left.+e^{i k_{z, i}\left|z-z^{\prime}\right|}\right) J_{0}\left(k_{r} r\right) k_{r} d k_{r} \\
G_{i+1}\left(\mathbf{R} ; \mathbf{R}^{\prime}\right) & =\int_{0}^{\infty} D e^{-i k_{z, i+1}(z-d)} J_{0}\left(k_{r} r\right) k_{r} d k_{r}
\end{aligned}
$$

where $k_{z, i}=\left(\left\langle k_{i}\right\rangle^{2}-k_{r}^{2}\right)^{1 / 2}$ is the vertical wavenumber in the $i$ th layer. The unknown constants, $A, B, C, D$, may be obtained by invoking the boundary conditions, leading to a system of linear equations which may be solved by the existing software such as Mathematica [29]. The 
solutions may be shown to be:

$$
\begin{aligned}
& B=\left(k_{z, i} \rho_{i-1}+k_{z, i-1} \rho_{i}\right) \frac{\mathcal{D}_{1}}{\mathcal{D}} \\
& C=e^{i k_{z, i} d}\left(k_{z, i+1} \rho_{i}+k_{z, i} \rho_{i+1}\right) \frac{\mathcal{D}_{2}}{\mathcal{D}}
\end{aligned}
$$

with $\mathcal{D}, \mathcal{D}_{1}$, and $\mathcal{D}_{2}$ given by

$$
\begin{aligned}
& \mathcal{D}=k_{z, i} k_{z, i+1} \rho_{i}+e^{2 i k_{z, i} d} k_{z, i} k_{z, i+1} \rho_{i-1} \rho_{i} \\
& -k_{z, i-1} k_{z, i+1} \rho_{i}^{2}+e^{2 i k_{z, i} d} k_{z, i-1} k_{z, i+1} \rho_{i}^{2} \\
& -k_{z, i}^{2} \rho_{i-1} \rho_{i+1}+e^{2 i k_{z, i} d} k_{z, i}^{2} \rho_{i-1} \rho_{i+1} \\
& +k_{z, i-1} k_{z, i} \rho_{i} \rho_{i+1}+e^{2 i k_{z, i} d} k_{z, i-1} k_{z, i} \rho_{i} \rho_{i+1} \\
& \mathcal{D}_{1}=-e^{i\left(k_{z, i} d+k_{z, i}\left|d-z^{\prime}\right|\right)} k_{z, i+1} \rho_{i}+\left.e^{i k_{z, i} \mid z^{\prime}}\right|_{z, i+1} \rho_{i} \\
& -e^{i\left(k_{z, i} d+k_{z, i}\left|d-z^{\prime}\right|\right)} k_{z, i} \rho_{i+1}-e^{i k_{z, i} \mid z^{\prime}} k_{z, i} \rho_{i+1} \\
& \left.D_{2}=-e^{i k_{z, i} \mid d-z^{\prime}}\left|k_{z, i} \rho_{i-1} e^{i\left(k_{z, i} d+k_{z, i} \mid z^{\prime}\right.}\right|\right) k_{z, i} \rho_{i-1} \\
& +e^{i k_{z, i} \mid d-z^{\prime}} \mid k_{z, i-1} \rho_{i}-e^{i\left(k_{z, i} d+k_{z, i}\left|z^{\prime}\right|\right) k_{z, i-1} \rho_{i}}
\end{aligned}
$$

A similar derivation may be applied to obtain the Green's function for the semi-infinite random medium, and in fact it is much less involved. For example, the Green's function for the lower most half-space, represented by $G_{N}$, can be expressed as:

$$
\begin{aligned}
G_{N}\left(\mathbf{R} ; \mathbf{R}^{\prime}\right) & =\int_{0}^{\infty} \frac{i}{k_{z, N}}\left(e^{i k_{z, N}\left|z-z^{\prime}\right|}\right. \\
& \left.+\mu e^{i k_{z, N}\left|z^{\prime}\right|-i k_{z, N} z}\right) J_{0}\left(k_{r} r\right) k_{r} d k_{r}
\end{aligned}
$$

where $\mu$ is the reflection coefficient given by

$$
\mu=\frac{\rho_{N-1} k_{z, N}-\rho_{N} k_{z, N-1}}{\rho_{N-1} k_{z, N}+\rho_{N} k_{z, N-1}}
$$

With the Green's function derived above, Equation (11) is an integro-differential equation whose complexity depends upon the spatial correlation of the medium randomness, represented by $\left\langle\mathrm{n}_{i}\left(\mathbf{R}^{\prime}\right) \mathrm{n}_{i}(\mathbf{R})\right\rangle$. In the most general case, the solution may only be possible using numerical method. However, in some specific cases, a semi-analytic solution may be obtained, one of which is to be discussed in a later section.

\section{Coherent reflection from rough surfaces}

Once the mean-field solutions for various layers are obtained, it is appropriate to consider the effects of surface roughness on wave propagation. It is conceivable that the mean-field solution in each layer consists of a particular solution corresponding to the non-homo- geneous term on the right-hand-side, and a homogeneous solution containing unknown constants, which are awaiting for further determination. These unknown constants may be determined by employing the boundary conditions at each interface, resulting in a global system of linear equations, which then are solved for the unknowns. For the case of flat interfaces, the above procedure is straightforward, however, for rough interfaces, it requires further developments, which fall into the area of rough surface scattering.

One of the standard treatments for scattering from randomly rough surfaces is the method of small perturbation, which is suitable for the case when the roughness is small compared with the acoustic wavelength, and the magnitude of the gradient of the roughness is much less than one [3].

Under the framework of perturbation analysis, the effect of roughness may be represented by several operators which in effect are to account for surface elevation and orientation. In this regard, the formulation developed by Kuperman and Schmidt [18], for the case of scattering from rough interfaces in a horizontallystratified media such as the present analysis, was found to be most convenient in terms of formula presentation and numerical computation.

According to the procedure for KupermanSchmidt's formulation, first, it is necessary to establish the linear system for the unknown amplitudes of wave components in various layers for the case of flat interfaces. With respect to this, it may be shown that, by applying the boundary conditions, the linear system may be conveniently expressed as

$$
\mathcal{B}\left(k_{r, 0}\right)<\chi\left(k_{r, 0}\right)>=C\left(k_{r, 0}\right)
$$

where $\mathcal{B}$ is the coefficients of the unknown amplitudes, and $\langle\chi\rangle$ is a vector containing the unknowns in each layer, and $C$ is for the source-related terms; the argument $k_{r, 0}$ represents the horizontal wavenumber of the incident plane wave.

Next, Equation (30) must be modified to account for surface roughness. This may be furnished by employing the boundary-perturbation analysis; the essence of which is to conduct a series of perturbation expansion, along with a sequence of algebraic manipulations associated with Fourier representation in order to arrive at a facilitating formulation. The process of derivation is shown in Ref. [18], and the resulting formulation is as follows:

$$
\left(\mathcal{B}\left(k_{r, 0}\right)+\frac{\left\langle\zeta_{i}^{2}\right\rangle}{2} \frac{\partial^{2} \mathcal{B}\left(k_{r, 0}\right)}{\partial z^{2}}+\mathcal{L}\left(k_{r, 0}\right)\right)\left\langle\chi\left(k_{r, 0}\right)\right\rangle=C\left(k_{r, 0}\right)
$$


in which the operator $\mathcal{L}$ is defined as

$$
\begin{aligned}
\mathcal{L}\left(k_{r, 0}\right) & =-\frac{\left\langle\zeta_{i}^{2}\right\rangle}{\sqrt{2 \pi}} \int d q P_{i}(q \\
& \left.-k_{r, 0}\right)\left(\frac{\partial \mathcal{B}(q)}{\partial z}-i\left(k_{r, 0}-q\right) \mathcal{B}_{s}(q)\right) \\
& \times \mathcal{B}^{-1}(q)\left(\frac{\partial \mathcal{B}\left(k_{r, 0}\right)}{\partial z}-i\left(q-k_{r, 0}\right) \mathcal{B}_{s}\left(k_{r, 0}\right)\right)
\end{aligned}
$$

where the matrix $\mathcal{B}_{s}$ stands for the effect of boundary orientation, and $P_{i}$ is the roughness power spectrum of the $i$ th interface. In comparison between Equations (30) and (31), it is found that the second and the third terms in Equation (31) are newly-developed operators accounting for rough surface elevation and rotation; examples for these operators shall be given in the following section.

\section{COHERENT REFLECTION FROM ROUGH SEABED: A CANONICAL MODEL}

The above formulation is completely general in nature, in terms of the dimensionality of surface roughness and/or the distribution of medium randomness. However, it is clear from Equations (11) and (31) that, for higher-dimensional rough surfaces and/or arbitrary distribution of medium inhomogenieties, the computational effort required to obtain the complete solution for the mean field and subsequently that for the coherent reflection is conceivably too immense to fulfill, so that appropriate simplification becomes necessary. Here, we shall consider a canonical problem representing a simplified version of of the seabed environment, i.e., a sediment layer overlying a rough seabottom, both assumed to be fluid medium for simplicity, as shown in Figure 2. Although artifactitiously simple, this model is suitable for the simulation of plane-wave interaction with an unconsolidated seabed, within which the shear modulus is less significant.

Furthermore, the two rough surfaces representing water/sediment and sediment/seabot-tom are assumed to be one-dimensional, with random elevation $z=\zeta_{i}(x)$. On the other hand, both the sediment layer and the seabottom are assumed to be anisotropic, with high correlation in the horizontal direction and low correlation in the vertical direction, so that the correlation function may be expressed as $[14,30]$ :

$$
\left\langle n_{i}(\mathbf{R}) n_{i}\left(\mathbf{R}^{\prime}\right)\right\rangle=4 \sigma_{i}^{2} N_{i}(\dot{r}) M_{i}(\dot{z}),(i=2,3)
$$

where $\sigma_{i}$ represents RMS randomness of $c_{i}^{\prime} /\left\langle c_{i}\right\rangle$, and $\dot{r}=\left|\mathbf{r}_{1}-\mathbf{r}_{2}\right|, \quad \dot{z}=\left|z_{1}-z_{2}\right| ; \quad N_{i}(\dot{r}) \quad$ and $\quad M_{i}(\dot{z})$ a re, respectively, the horizontal and vertical correlation func-

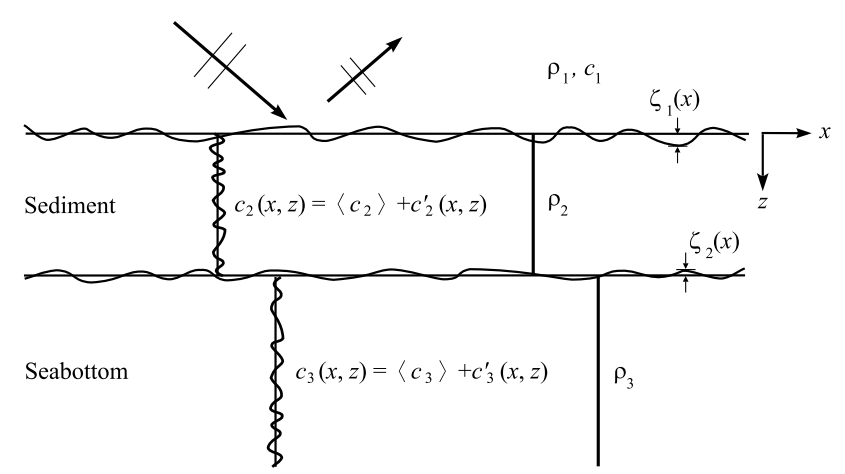

Fig. 2. A simplified version of seabed environment.

tions of the random sound-speed variations.

Substituting Equation (33) into Equation (11), the equation governing the mean field becomes

$$
\begin{aligned}
& \nabla^{2}\left\langle p_{i}\right\rangle+\left\langle k_{i}\right\rangle^{2}\left\langle p_{i}\right\rangle=\frac{\left\langle k_{i}\right\rangle^{4} \sigma_{i}^{2}}{\pi} \int_{V\left(\mathbf{R}^{\prime}\right)} N_{i}\left(\dot{r}^{\prime}\right) M_{i}\left(\bar{z}^{\prime}\right) \\
& \left\langle p_{i}\left(\mathbf{R}^{\prime}\right)\right\rangle G_{i}\left(\mathbf{R} ; \mathbf{R}^{\prime}\right) d V\left(\mathbf{R}^{\prime}\right)
\end{aligned}
$$

For practical purposes, the horizontal and vertical correlations are taken to be Gaussian and Dirac-delta function, respectively, i.e.,

$$
\begin{aligned}
& N_{i}(\dot{r})=e^{-\dot{r}^{2} / L Z_{, i}} \\
& M_{i}(\dot{z})=z_{0, i} \delta(\bar{z})
\end{aligned}
$$

where $L_{0, i}$ is the horizontal correlation distance for layer $i$, and $z_{0, i}$ is a constant.

In the sections followed, we shall first derive the mean-field solutions for the sediment layer $(i=2)$ and the seabottom $(i=3)$, and then formulate the coherent reflection from the rough seabed.

\section{Mean-field solutions in random sediment layer and seabottom}

Inserting Equation (36) into Equation (34), and considering the fact that the incoming plane wave lies on $x-z$ plane and the roughness is one-dimensional so that there shall have no out-of-plane wave component, the solution for $\left\langle p_{i}\right\rangle$, being independent of $y$, may be expressed as

$$
\left\langle p_{i}(x, z)\right\rangle=e^{-i k_{r, 0} x} q_{i}(z), \quad(i=2,3)
$$

Substituting Equations (36) and (37) into Equation (34), and assuming that $z_{0, i}<<\lambda$, where $\lambda$ is the acoustic wavelength, Equation (34) may be analytically simplified to become [27] 


$$
\begin{aligned}
& {\left[\frac{d^{2}}{d z^{2}}+\left(\left\langle k_{i}\right\rangle^{2}-k_{r, 0}^{2}\right)\right] q_{i}(z)=-2\left\langle k_{i}\right\rangle^{4} \sigma_{i}^{2} z_{0, i} q_{i}(z) \int_{0}^{\infty} N_{i}(\xi)} \\
& J_{0}\left(k_{r, 0} \xi\right) G_{i}\left(\xi, z ; z^{\prime}\right)
\end{aligned}
$$

The above equation was derived under the assumptions that $q_{i}\left(z^{\prime}\right)$ and $G_{i}\left(x, z ; x^{\prime}, z^{\prime}\right)$ embedded in Equation (34) are slowly varying functions over $z^{\prime}$ so that it was set to be equal to $z$, and then subsequently taken out of integral with respect to $z^{\prime}$.

Based upon Equation (38), we are here to derive the mean-field solution in the random media. To further simply the formulation, with $z^{\prime}=z$, Equation (21) for $i$ $=2$ is rewritten as

$$
\begin{aligned}
G_{2}\left(\mathbf{R} ; \mathbf{R}^{\prime}\right) & =\int_{0}^{\infty}-\frac{1}{i k_{z, 2}}\left(B_{1} e^{2 i k_{z, 2} z}+C_{1} e^{-2 i k_{z, 2} z}\right. \\
& \left.+B_{2}+C_{2}+1\right) J_{0}\left(k_{r} r\right) k_{r} d k_{r}
\end{aligned}
$$

where

$$
\begin{aligned}
& B_{1}=\left(k_{z, 2} \rho_{1}+k_{z, 1} \rho_{2}\right)\left(k_{z, 3} \rho_{2}-k_{z, 2} \rho_{3}\right) \frac{1}{\mathcal{D}} \\
& B_{2}=-\left(k_{z, 2} \rho_{1}+k_{z, 1} \rho_{2}\right)\left(k_{z, 3} \rho_{i}+k_{z, 2} \rho_{3}\right) \frac{e^{2 i k_{z, 2} d}}{\mathcal{D}} \\
& C_{1}=\left(k_{z, 3} \rho_{i}+k_{z, 2} \rho_{3}\right)\left(k_{z, 1} \rho_{2}-k_{z, 2} \rho_{1}\right) \frac{e^{2 i k_{z, 2} d}}{\mathcal{D}} \\
& C_{2}=-\left(k_{z, 3} \rho_{2}+k_{z, 2} \rho_{3}\right)\left(k_{z, 1} \rho_{2}-k_{z, 2} \rho_{1}\right) \frac{e^{2 i k_{z, 2} d}}{\mathcal{D}}
\end{aligned}
$$

Further substitution of Equations (35) and (39) into Equation (38), and via some algebraic manipulations result in a nonhomogeneous second-order ordinary differential equation:

$$
\frac{d^{2} q_{2}}{d z^{2}}+\eta_{2}^{2} q_{2}=-\mathcal{F}_{2}(z) q_{2}
$$

where

$$
\begin{gathered}
\mathcal{F}_{2}(z)=2\left\langle k_{2}\right\rangle^{4} \sigma_{2}^{2} z_{0,2} \int_{0}^{\infty} \frac{i}{k_{z, 2}} \mathcal{H}_{2}\left(k_{r}, k_{r, 0}\right) \\
\left(B_{1} e^{2 i k_{z, 2} 2^{z}}+C_{1} e^{-2 i k_{z, 2}}\right) k_{r} d k_{r}
\end{gathered}
$$

with relevant parameters and functions defined as follows:

$$
\begin{aligned}
\eta_{2}^{2}=\left\langle k_{2}\right\rangle^{2}\left[1-\frac{k_{r, 0}^{2}}{\left\langle k_{2}\right\rangle^{2}}+c_{2}\left(k_{r, 0}\right)\right] \\
c_{2}\left(k_{r, 0}\right)=2\left\langle k_{2}\right\rangle^{2} \sigma_{2}^{2} z_{0,2} \int_{0}^{\infty} \frac{i}{k_{z, 2}} H_{2}\left(k_{r}, k_{r, 0}\right)\left(B_{2}\right. \\
\left.+C_{2}+1\right) k_{r} d k_{r}
\end{aligned}
$$

$$
\begin{aligned}
\mathcal{H}_{2}\left(k_{r}, k_{r, 0}\right) & =\int_{0}^{\infty} N_{2}(\xi) J_{0}\left(k_{r, 0} \xi\right) J_{0}\left(k_{r} \xi\right) \xi d \xi \\
& =\frac{1}{2} L_{0,2}^{2} e^{-\left(k_{r, 0}^{2}+k_{r}^{2}\right) L \gamma_{, 2} / 4} I_{0}\left(k_{r, 0} k_{r} L_{0,2}^{2}\right.
\end{aligned}
$$

Due to the fact that $\left|\mathcal{F}_{2}(z)\right|<<\left|\eta_{2}^{2}\right|$, Equation (44) may be solved by first dropping the right-hand-side to obtain the first-iteration solution, and then solving the full equation by the method of variation of parameter [2] to yield:

$$
q_{2}(z)=\left\langle A_{1}\right\rangle y_{1}(z)+\left\langle A_{2}\right\rangle y_{2}(z)
$$

where

$$
\begin{aligned}
y_{1}(z) & =e^{-i \eta_{2} z}-\frac{1}{2 i \eta_{2}}\left[e^{-i \eta_{2} z} \int_{z}^{0} \mathcal{F}_{2}\left(z^{\prime}\right) d z^{\prime}\right. \\
& \left.+e^{i \eta_{2} z} \int_{d}^{z} \mathcal{F}_{2}\left(z^{\prime}\right) e^{-2 i \eta_{2} z^{\prime}} d z^{\prime}\right] \\
y_{2}(z) & =e^{i \eta_{2} z}-\frac{1}{2 i \eta_{2}}\left[e^{-i \eta_{2} z} \int_{z}^{0} \mathcal{F}_{2}\left(z^{\prime}\right) e^{2 i \eta_{2} z^{\prime}} d z^{\prime}\right. \\
& \left.+e^{i \eta_{2} z} \int_{d}^{z} \mathcal{F}_{2}\left(z^{\prime}\right) d z^{\prime}\right]
\end{aligned}
$$

So, the desired solution for the mean-field inside the sediment layer is

$$
\left\langle p_{2}(x, z)\right\rangle=\left(\left\langle A_{1}\right\rangle y_{1}(z)+\left\langle A_{2}\right\rangle y_{2}(z)\right) e^{-k_{r, 0} x}
$$

A similar procedure may be applied to obtain the mean-field solution for the seabottom half-space, and the results may be shown to be:

$$
\begin{aligned}
& \left\langle p_{3}(x, z)\right\rangle=\langle\mathcal{T}\rangle\left[e^{-i \eta_{3}(z-d)}-\frac{e^{i \eta_{3}(z-d)}}{2 i \eta_{3}}\right. \\
& \left.\left(\int_{-\infty}^{z-d} \mathcal{F}_{3}(\xi) e^{-i 2 \eta_{3} \xi} d \xi-\frac{e^{-i \eta_{3}(z-d)}}{2 i \eta_{3}}\right)\right] e^{-i k_{r, 0} x}
\end{aligned}
$$

where

$$
\begin{gathered}
\mathcal{F}_{3}(\xi)=2\left\langle k_{3}\right\rangle^{4} \sigma_{3}^{2} z_{0,3} \int_{0}^{\infty} \frac{i \mu_{32}}{k_{z, 3}} e^{-i 2 k_{z, 3} \xi^{\xi} \mathcal{H}_{3}} \\
\left(k_{r}, k_{r, 0}\right) k_{r} d k_{r} \\
\eta_{3}^{2}=\left\langle k_{3}\right\rangle^{2}\left[1-\frac{k_{r, 0}^{2}}{\left\langle k_{3}\right\rangle^{2}}+c_{3}\left(k_{r, 0}\right)\right]
\end{gathered}
$$




$$
\begin{aligned}
& c_{3}\left(k_{r, 0}\right)=2\left\langle k_{3}\right\rangle^{2} \sigma_{3}^{2} z_{0,3} \int_{0}^{\infty} \frac{i}{k_{z, 3}} \mathcal{H}_{3}\left(k_{r}, k_{r, 0}\right) k_{r} d k_{r} \\
& \mathcal{H}_{3}\left(k_{r}, k_{r, 0}\right)=\frac{1}{2} L_{0,3}^{2} e^{-\left(k_{x, 0} x+k_{z, 1} z\right)}+\left\langle\mathcal{R}_{1}\right\rangle e^{-i\left(k_{x, 0} x-k_{z, 1} z\right)}
\end{aligned}
$$

Furthermore, since the upper half-space is homogeneous, their solutions may be expressed as

$$
p_{1}(x, z)=e^{-i\left(k_{x}, 0^{x+k}, 1^{z}\right)}+\left\langle\mathcal{R}_{1}\right\rangle e^{-i\left(k_{x, 0} x-k_{z, 1} z\right)}
$$

\section{Coherent reflection: boundary operators}

The coherent reflection may be obtained by solving the linear system shown in Equation (31). Here, we present the relevant operators such as $\mathcal{B}$ and its derivatives, and $\mathcal{B}_{s}$, for the specialized problem under consideration as shown in Figure 2. The derivation, although a bit of tedious, is rather straightforward.

The operator $\mathcal{B}$ may be derived from the meanfield solutions, Equations (53), (54), and (59) by employing the appropriate boundary conditions, yielding

$$
\begin{aligned}
& \mathcal{B}\left(k_{r, 0}\right)=\left[\begin{array}{cc}
1 & -\frac{1}{2 i \eta_{2}}\left(2 i \eta_{2}-h_{21}\right) \\
\frac{i k_{z, 1}}{\rho_{1} \omega^{2}} & \frac{1}{2 \rho_{2} \omega^{2}}\left(2 i \eta_{2}+h_{21}\right) \\
0 & -\frac{e^{-i \eta_{2} d}}{2 i \eta_{2}}\left(2 i \eta_{2}-h_{22}\right) \\
0 & -\frac{e^{-i \eta_{2} d}}{2 \rho_{2} \omega^{2}}\left(2 i \eta_{2}-h_{22}\right)
\end{array}\right. \\
& -\frac{1}{2 i \eta_{2}}\left(2 i \eta_{2}-h_{22}\right) \\
& -\frac{1}{2 \rho_{2} \omega^{2}}\left(2 i \eta_{2}-h_{22}\right) \quad 0 \\
& \frac{e^{i \eta_{2} d}}{2 i \eta_{2}}\left(2 i \eta_{2}-h_{23} e^{-2 i \eta_{2} d}\right) \quad-1+\frac{h_{3}}{2 i \eta_{3}} \\
& \frac{e^{i \eta_{2} d}}{2 \rho_{2} \omega^{2}}\left(2 i \eta_{2}+h_{23} e^{-2 i \eta_{2} d}\right) \frac{1}{2 \rho_{3} \omega^{2}}\left(2 i \eta_{3}+h_{3}\right) \\
& \chi=\left[\langle\mathcal{R}\rangle,\left\langle A_{1}\right\rangle,\left\langle A_{2}\right\rangle,\langle\mathcal{T}\rangle\right]^{T} \\
& C=\left[-1, \frac{i k_{z, 1}}{\rho_{1} \omega^{2}}, 0,0\right]^{T}
\end{aligned}
$$

with $h_{21}, h_{22}$ and $h_{23}, h_{3}$, being function of $k_{r, 0}$, defined as follows:

$$
\left[\begin{array}{l}
h_{21} \\
h_{22} \\
h_{23}
\end{array}\right]=\int_{d^{\prime}}^{0} \mathcal{F}_{2}\left(z^{\prime}\right)\left[\begin{array}{c}
e^{-2 i \eta_{2} z^{\prime}} \\
1 \\
e^{2 i \eta_{2} z^{\prime}}
\end{array}\right] d z^{\prime}
$$

$$
=\int_{0}^{\infty} \mathcal{H}_{2}^{*}\left(k_{r}, k_{r, 0}\right)\left[\begin{array}{c}
B_{1} \frac{e^{2 i d\left(k_{z, 2}-\eta_{2}\right)-1}}{k_{z, 2}-\eta_{2}}-C_{1} \frac{e^{-2 i d\left(k_{z, 2}+\eta_{2}\right)}-1}{k_{z, 2}+\eta_{2}} \\
B_{1} \frac{e^{2 i k k_{z, 2} 2_{-1}}}{k_{z, 2}}-C_{1} \frac{e^{-2 i k k_{z, 2} 2_{-1}}}{k_{z, 2}} \\
B_{1} \frac{e^{2 i d\left(k_{z, 2}+\eta_{2}\right)-1}}{k_{z, 2}+\eta_{2}}-C_{1} \frac{e^{-2 i d\left(k_{z, 2}-\eta_{2}\right)-1}}{k_{z, 2}-\eta_{2}}
\end{array}\right] k_{r} d k_{r}
$$

$$
\begin{aligned}
& h_{3}=\int_{-\infty}^{0} F_{3}\left(z^{\prime}\right) e^{-i 2 \eta_{3} z^{\prime}} d z^{\prime} \\
& =\int_{0}^{\infty} \mathcal{H}_{3}^{*}\left(k_{r}, k_{r, 0}\right) \frac{\mu_{32}}{k_{z, 3} \eta_{3}} k_{r} d k_{r}
\end{aligned}
$$

where $\mathcal{H}_{3}^{*}\left(k_{r}, k_{r, 0}\right)=-\frac{\left\langle k_{i}\right\rangle^{4} \sigma_{i}^{2} z_{0, i}}{k_{z, i}} \mathcal{H}_{i}\left(k_{r}, k_{r, 0}\right), \quad(i=1,2)$. Furthermore, the operators $\frac{\partial \mathcal{B}}{\partial z}\left(k_{r, 0}\right)$ and $\frac{\partial^{2} \mathcal{B}}{\partial z^{2}}\left(k_{r, 0}\right)$, appeared in Equations (31) and (32), may be obtained from the mean-field solutions by differentiating with respect to $z$, and then rearranging with an order corresponding to coefficient matrix, Equation (60), in the linear system. The results may be shown to be:

$$
\frac{\partial \mathcal{B}}{\partial z}\left(k_{r, 0}\right)=\left[\begin{array}{cc}
i k_{z, 1} & i \eta_{2}+\frac{h_{21}}{2} \\
-\frac{k_{z, 1}^{2}}{\rho_{1} \omega^{2}} & \frac{1}{\rho_{2} \omega^{2}}\left(\eta 8+\frac{i \eta_{21} h_{21}}{2}+\mathcal{F}_{2}(0)\right. \\
0 & e^{-i \eta_{2} d}\left(-i \eta_{2}+\frac{h_{22}}{2}\right) \\
0 & \frac{e^{-i \eta_{2} d}}{\rho_{2} \omega^{2}}\left(-\eta_{2}-\frac{i \eta_{2} h_{22}}{2}-\mathcal{F}_{2}(d)\right)
\end{array}\right.
$$

$$
-i \eta_{2}+\frac{h_{22}}{2}
$$

$\frac{1}{\rho_{2} \omega^{2}}\left(\eta \eta_{2}+\frac{i \eta_{2} h_{22}}{2}+\mathcal{F}_{2}(0)\right.$

$$
e^{\left.i \eta_{3} d_{\left(i \eta_{2}\right.}+\frac{i \eta_{2} h_{22}}{2}+\mathcal{F}_{2}(0)\right)}
$$$$
i \eta_{3}+\frac{h_{3}}{2}
$$$$
\frac{e^{i \eta_{2} d}}{\rho_{2} \omega^{2}}\left(-\eta_{2}-\frac{i \eta_{1} e^{-i 2 \eta_{2} d h_{22}}}{2}\right) \quad \frac{1}{\rho_{3} \omega^{2}}\left(\eta_{3}^{2}+\frac{i \eta_{3} h_{3}}{2}+\mathcal{F}_{3}(0)\right.
$$

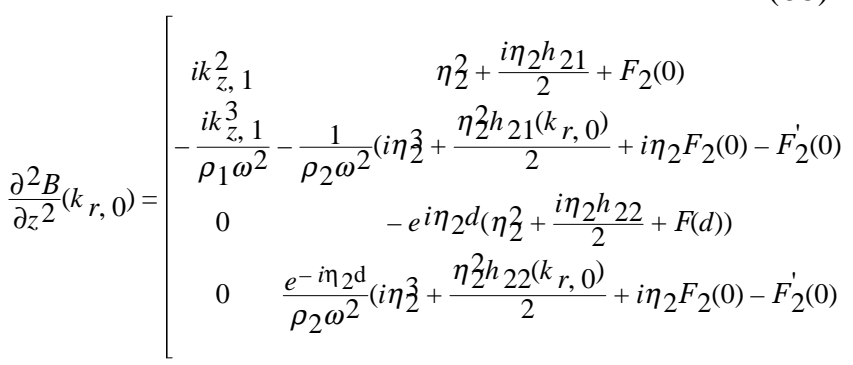




$$
\begin{aligned}
& \eta_{2}+\frac{i \eta_{2} h_{22}}{2}+\mathcal{F}_{2}(0) \\
& -\frac{1}{\rho_{2} \omega^{2}}\left(i \eta_{2}^{3}+\frac{\eta \hat{2}_{22} h_{2}(k, 0)}{2}+i \eta_{2} \mathcal{F}_{2}(0)-\mathcal{F}_{2}^{\prime}(0)\right. \\
& -e^{i \eta_{2} d}\left(\eta_{2}+\frac{i \eta_{2} h_{23}}{2}+\mathcal{F}(d)\right) \\
& \frac{e^{i \eta} 2^{\mathrm{d}}}{\rho_{2} \omega^{2}}\left(-i \eta \underline{z}+\frac{\eta 2_{2} h_{23}(k r, 0)}{2}+i \eta_{2} \mathcal{F}_{2}(0)-\mathcal{F}_{2}^{\prime}(0)\right. \\
& 0 \\
& 0 \\
& \eta_{3}^{2}+\frac{i \eta_{3} h_{3}}{2}+\mathcal{F}(0) \\
& -\frac{1}{\rho_{2} \omega^{2}}\left(i \eta \frac{\eta}{3}+\frac{\eta h_{3} h_{3}}{2}+i \eta_{3} \mathcal{F}_{3}(0)-\mathcal{F}_{3}^{\prime}(0)\right)
\end{aligned}
$$

The matrix $\mathcal{B}_{s}\left(k_{r, 0}\right)$ represents the effect of surface orientation of the rough surface, which in reference to the general formulation given in Reference [18] may be shown to yield the following result for the present case:

$$
\begin{aligned}
& \mathcal{B}_{x}\left(k_{r, 0}\right)=\frac{i k_{r, 0}}{\rho_{2} \omega^{2}}\left[\begin{array}{cc}
0 & 0 \\
\frac{\rho_{2}}{\rho_{1}} & -1+\frac{h_{21}}{2 i \eta_{2}} \\
0 & 0 \\
0 & e^{-i \eta_{2} d_{-}} \frac{e^{-i \eta_{2} d h_{22}}}{2 i \eta_{2}}
\end{array}\right. \\
& \left.\begin{array}{cc}
0 & 0 \\
-1+\frac{h_{22}}{2 i \eta_{2}} & 0 \\
0 & 0 \\
e^{-i \eta_{2} d-\frac{e^{-i \eta_{2} d h_{23}}}{2 i \eta_{2}}}-\frac{\rho_{2}}{\rho_{1}}\left(1-\frac{h_{3}}{2 i \eta_{3}}\right)
\end{array}\right]
\end{aligned}
$$

Finally, the power spectrum for the rough surface embedded in Equation (32) is also taken to be Gaussian for convenience:

$$
P_{i}(k)=\sqrt{2 \pi} \ell_{i} e^{-k^{2} \ell_{i}^{2} / 2},(i=1,2)
$$

Here, $\ell_{i}$ represents the correlation length of the roughness. The linear system, Equation (31), may now be solved for $\langle R\rangle$ to obtain the coherent reflection coefficient.

\section{RESULTS AND DISCUSSION}

In this section, we shall employ the above formulations and generate numerical results for the reflection coefficient. Since the incident wave is taken to be a unit amplitude, so that the parameter $\langle\mathcal{R}\rangle$ is the reflection coefficient. It is noted that $\langle R\rangle$ stands for the ensemble average of the reflected field, therefore it represents the coherent component.
There are many parameters involved in this problem, including averaged acoustic properties of the media, $\rho_{i},\left\langle c_{i}\right\rangle$, surface-roughness properties, $\zeta_{i}, \ell_{i}$; medium-randomness properties, $z_{0, i}, L_{0, i}$; frequency $f$ and sediment thickness $d$. Since the parameters are too many to make a complete analysis for every one of them, we only choose those characterizing the surface roughness and medium randomness to examine their effects on the coherent reflection coefficient. A combined parameter, defined as $k d$, where $k=2 \pi f / \mathrm{c}$ is the acoustic wavenumber, representing the ratio of sediment thickness and wavelength (thickness-to-wavelength ratio, for short) is a controlling parameter of this analysis. Unless otherwise restated in the text or in the legends of figures, the following parameters are chosen as given below: $c_{1}=1500 \mathrm{~m} / \mathrm{s}, \rho_{1}=1000 \mathrm{~kg} / \mathrm{m}^{3} ;\left\langle c_{2}\right\rangle=$ $1800 \mathrm{~m} / \mathrm{s}, \rho_{2}=1300 \mathrm{~kg} / \mathrm{m}^{3} ;\left\langle c_{3}\right\rangle=2000 \mathrm{~m} / \mathrm{s}, \rho_{3}=1500$ $\mathrm{kg} / \mathrm{m}^{3} ; L_{0,2}=L_{0,3}=5.0 \mathrm{~m} ; z_{0,2}=z_{0,3}=1.0 \mathrm{~m} ; \ell_{0,2}=\ell_{0,3}$ $=10 \mathrm{~m}$. It is to be noted that the values employed are merely intended to demonstrate the fundamental mechanisms of this analysis, so that they may not reflect the true values in a realistic environment, such as those data surveyed by Hamilton [12].

Figure 3 shows the result for $f=200 \mathrm{~Hz}, d=20 \mathrm{~m}$, (or $k d=16.75$, which represents a large value of thickness-to-wavelength ratio), and the surface roughness and medium randomness are chosen, respectively, as $\zeta_{1}$ $=\zeta_{1}=0.4 \mathrm{~m}, \sigma_{2}=\sigma_{3}=0.15$.

The figure shows that, for the case of flat interfaces and uniform media, the distribution of the reflection coefficient (dotted curve) oscillates and increases in average when the incident grazing angles decreases from normal incident toward shallower angles, and arrives at the value of unity as it reaches the critical angle, which in the present case is $\theta_{c}=\cos ^{-1}(1500 /$ $2000)=41.4^{\circ}$. The oscillatory behavior is obviously due to multiple reflection inside the sediment layer.

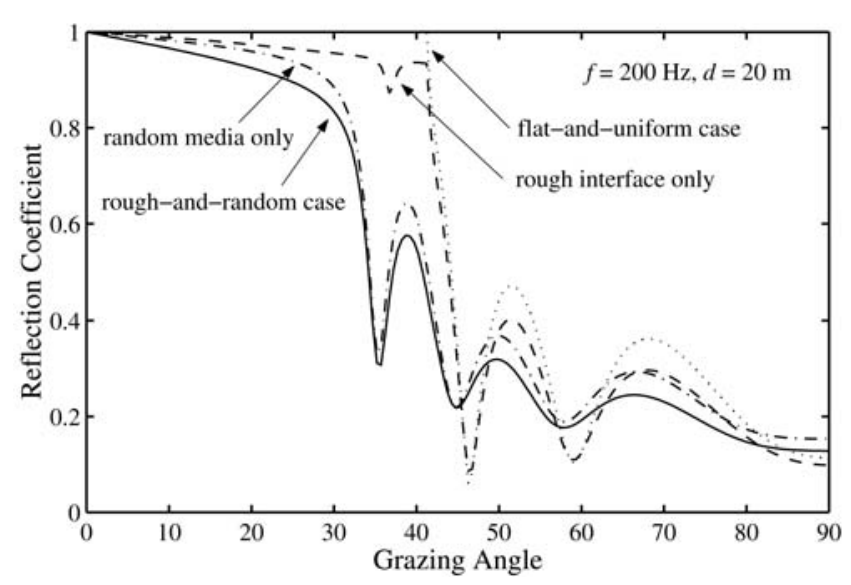

Fig. 3. Reflection coefficient for $f=200 \mathrm{~Hz}, d=20 \mathrm{~m}$. 
If the interfaces are randomly rough, the result shown by the dashed curve illustrates that in general the coefficient is less than that for the smooth-and-uniform case. On the other hand, if the sound-speed distribution inside the media is subject to random perturbation, the reflection coefficient (dashed-and-dotted curve) is also decreased, particularly in the total reflection region; both results show that there exists no total reflection. This is readily understood in that either interface roughness or medium inhomogenieties shall develop a scattering field, extracting part of coherent energy and then spreading into various non-specular angles; subsequently, the coherent reflection coefficients are reduced. The overall effect of both surface roughness and medium inhomogenieties are shown by the solid curve, which indicates that the reduction is larger than each individual. It is interesting to notice that the behavior of the distributions between the two curves are distinctively different in the neighborhood of the critical angle, indicating that the variation of overall curve near the critical angle offers a potential means for identifying the dominant scattering mechanism of the problem.

Figure 4 shows the results for a lower frequency $f$ $=125 \mathrm{~Hz}$, and the rest of the parameters are the same as those for Figure 3. It is seen that as the frequency becomes lower the difference between the curve for the flat-and-uniform case and those for other cases reduces, indicating that the significance of the scattering effects becomes less. This is due to the fact that for lower frequency the relative magnitudes of surface roughness and medium randomness with respect to wavelength are smaller, which in turn reduce the intensity of the scattering field. It is expected that if the frequency continues to decrease, the scattering field shall diminish, and eventually all curves tend to overlap, as shown in Figure 5 for $f=75 \mathrm{~Hz}$.

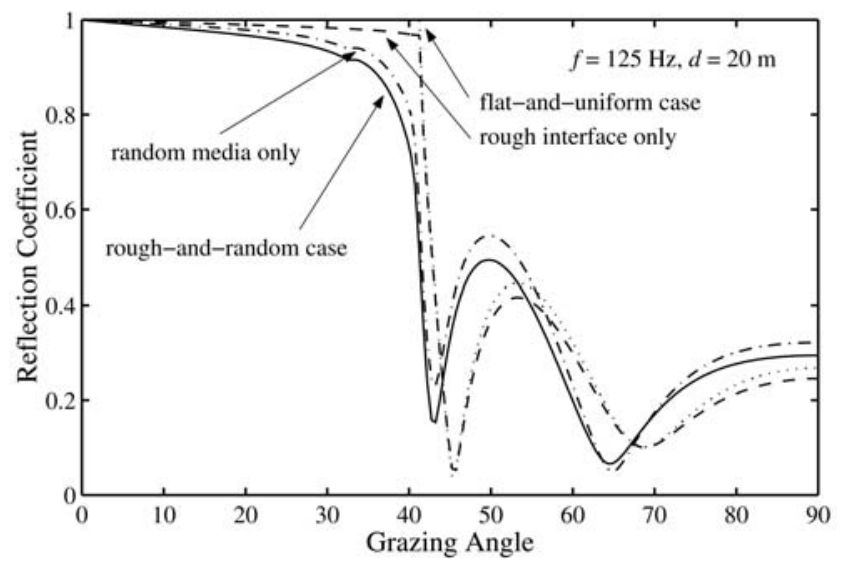

Fig. 4. Reflection coefficient for $f=125 \mathrm{~Hz}, d=20 \mathrm{~m}$.

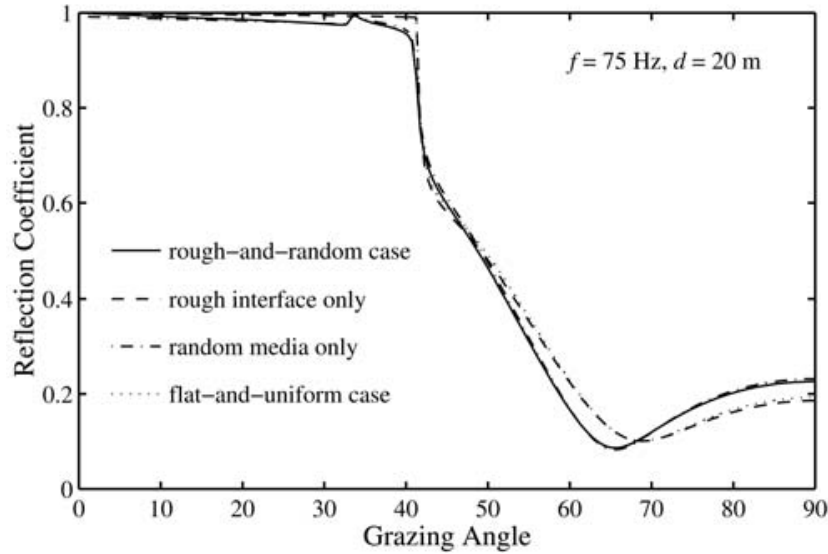

Fig. 5. Reflection coefficient for $f=75 \mathrm{~Hz}, d=20 \mathrm{~m}$

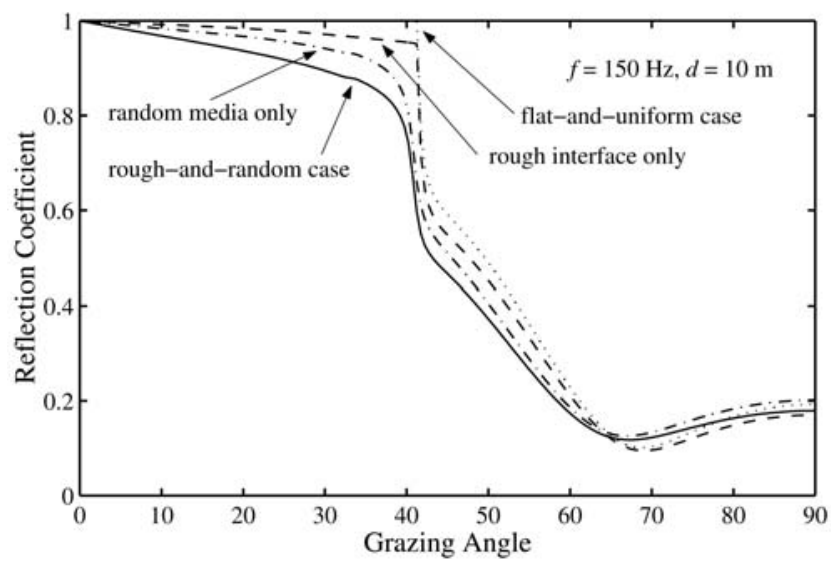

Fig. 6. Reflection coefficient for $f=150 \mathrm{~Hz}, d=10 \mathrm{~m}$

As mentioned previously, the combination of $k d$ is a controlling parameter which measures the significance of sediment layer. When $k d$ is large, the properties of the sediment layer will dominate the scattering effect, for the wave is unable to sense the properties of the bottom layer. However, it is to be noticed that the same values of $k d$ does not guarantee to have the same results. Figure 6 shows the result for $f=150 \mathrm{~Hz}$ and $d$ $=10 \mathrm{~m}$, which apparently has the same value of $k d$ as that for Figure 5, but the two results show a clear difference. For the later case, the thickness is thin enough so that the bottom layer begins to play effect, and when it is compared with Figure 3, it is conceivable that the multiple reflection inside the sediment layer becomes less significance.

Figure 7 examines the effect of medium inhomogenieties on the reflection coefficient. The parameters employed are the same as those in Figure 3, except that the parameter $\sigma_{2}$ varies to yield three curves 


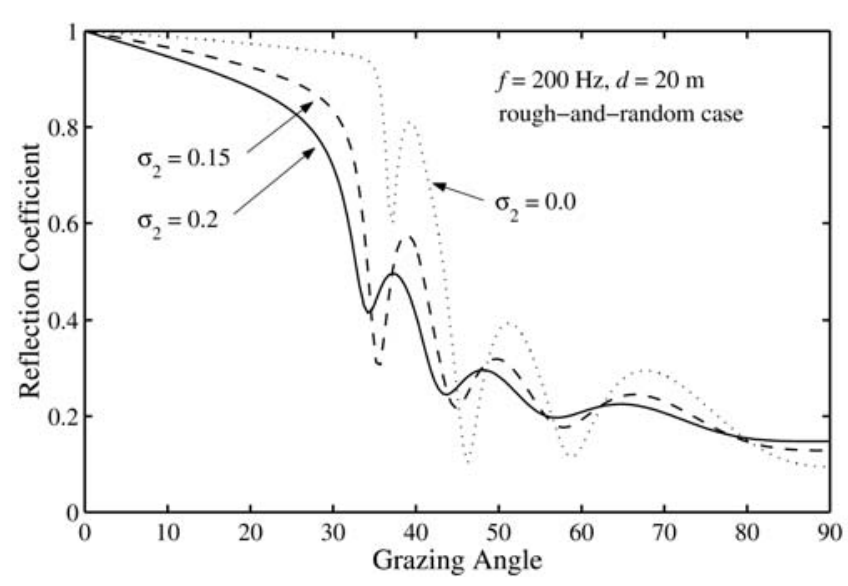

Fig. 7. Effect of $\sigma_{2}$ on reflection coefficient for $f=200 \mathrm{~Hz}, d=20 \mathrm{~m}$.

represented by dotted, dashed, and solid lines, corresponding to $\sigma_{2}=0.0,0.15,0.2$, respectively. The results show that the degree of randomness inside the sediment layer significantly affect the reflection coefficient, with the expected variations that the higher degree of randomness (represented by larger $\sigma_{2}$ ) the lower the coherent reflection. If the same situation is applied to the seabottom layer, the result is shown in Figure 8. It is seen that the increment of $\sigma_{3}$ in this case only produces a small effect near the area of critical angle. Of course, this does not imply that the randomness in the seabottom layer is immaterial, it simply indicates that for the set of parameters chosen, in particular, large $k d(k d=16.75)$, the variation of seabottom layer is relatively insensitive. When $k d$ is small due to thin sediment layer and relatively high frequency, then the parameter $\sigma_{3}$ should be expected to be significant.

\section{CONCLUSIONS}

This paper considers the coherent reflection of an acoustic plane wave from rough interfaces in a horizontally-stratified random fluid medium. The general formulation incorporating the general theory for wave scattering from stratified media and that for scattering in random media is derived, and then applied to an oceanic environment to study their effects on the coherent reflection. Many efforts are spent to derive the Green's function in a random slab, which constitutes a generic formulation of the problem.

Many numerical results for a simplified version of seabed environmental model are generated and analyzed. It has been found that due to the generation of scattering field, attributed to either the surface roughness or medium inhomogenieties. the coherent reflection decreases to some extent depending upon the degree of randomness.

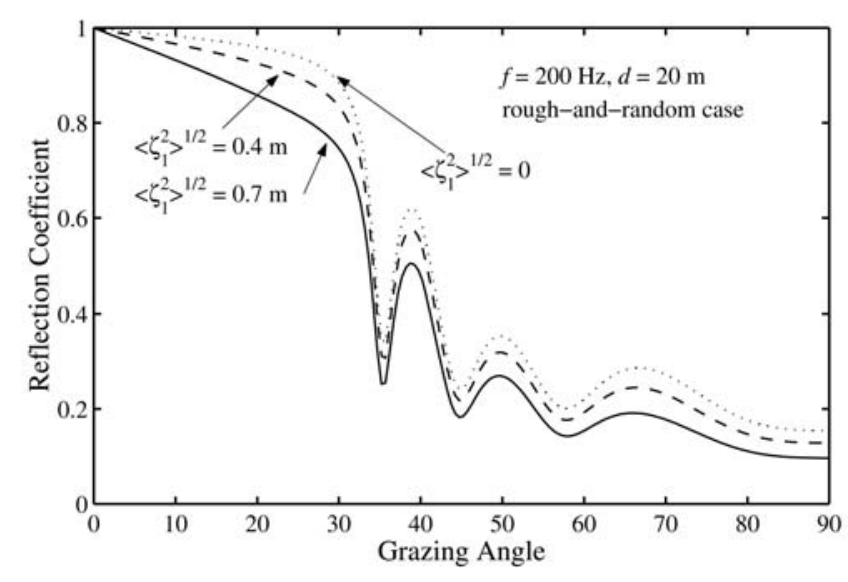

Fig. 8. Effect of $\sigma_{3}$ on reflection coefficient for $f=200 \mathrm{~Hz}, d=20 \mathrm{~m}$.

These findings are consistent with the general theory of scattering, in that the scattering field acts to distract energy from specular reflection, resulting in a loss of the coherent energy.

It is also interesting to note that the variation of the distribution for the coherent reflection as function of the incident grazing angle shows a distinct characteristic with respect to scattering mechanisms, particularly in the neighborhood of the critical angle. This feature may be served as a basis for identifying the dominant scattering mechanisms in the problem.

To further current study, it is possible to include the consideration of the density perturbation. Moreover, the averaged acoustic properties, such as $\left\langle c_{i}\right\rangle$ and $\left\langle\rho_{i}\right\rangle$, in general vary with respect to depth coordinate. Also, the scattering field for the problem under consideration is also an important issue. These problems shall be investigated in the ensuing study, with the foundation laid by the present analysis.

\section{ACKNOWLEDGEMENT}

This work was supported in part by National Science Council of Taiwan, R.O.C. through contracts NSC89-2611-M-110-012 and NSC89-2611-E-110-021. The authors would like to express our profound thanks for their financial supports.

\section{REFERENCES}

1. Bergamann, P.G. "The wave equation in a medium with a variable index of reflection," J. Acoust. Soc. Am., 17 (4), 329-333 (1964).

2. Boyce, W.E. and DiPrima, R.C., Elementary Differential Equations and Boundary Value Problems, 6th edi., John Wiley (1997). 
3. Brekhovkikh, L.M. and Lysanov, Y.P., Fundamentals of Ocean Acoustics, 2nd edi., Springer-Verlag (1991).

4. Brekhovkikh, L.M., Waves in Layered Media, 2nd edi. (translated by R.T. Beyer), Academic Press (1980).

5. Chernov, L.A., Wave Propagations in a Random Medium, McGraw-Hill, New York (1960).

6. Dozier, L.B. and Tappert, F.D., "Statistics of normal mode amplitudes in a random ocean: Theory," J. Acoust. Soc. Am., 63, 352-365, (1977).

7. Desaubies, Y., "On the scattering of sound by internal waves in the ocean," J. Acoust. Soc. Am., 64, 1460-1468 (1978).

8. Essen, H., Schirmer, F., and Sirkes, S., "Acoustic remote sensing of internal waves in shallow water," Int. J. Remote Sens. 4, 33-47 (1983).

9. Ewing, W.M., Jardetzky, W.S., and Press, F., Elastic Waves in Layered Media, McGraw-Hill (1957).

10. Frisk. G.V., "Inhomogeneous waves and the plane wave reflection coefficient," J. Acoust. Soc. Am. 66, 219-234 (1979).

11. Goff, J.A. and Jordan, T., "Stochastic modeling of seafloor morphology: Inversion of Sea Beam data for second-order statistics," J. Geophys. Res., 93, 13,589-13, 608 (1988).

12. Hamilton, H., "Geoacoustic modeling of the sea floor," J. Acoust. Soc. Am., 68 (5), 1313-1340 (1980).

13. Ishiruma, A., Wave Propagation and scattering in Random Media, Volume 1 and 2, Academic Press (1978).

14. Ivakin, A.N. and Lysanov, P., "Theory of underwater sound scattering by random inhomogenieties of the bottom,' Sov. Phys. Acoust, 27, 61-64 (1981).

15. Jensen, F.B., Kuperman, W.A., Porter, M.B., and Schmidt, H., Computational Ocean Acoustics, AIP Press (1994).

16. Kuperman, W.A., "Coherent component of specular reflection and transmission at a randomly rough twofluid interface,', J. Acoust. Soc. Am., 58, 365-370 (1975).

17. Kuperman, W.A., Schmidt, "Rough surface elastic wave scattering in a horizontally stratified ocean,' J. Acoust. Soc. Am. 79, 1767-1777 (1986).

18. Kuperman, W.A. and Schmidt, H., "Self-consistent perturbation approach to rough surface scattering in stratified elastic media," J. Acoust. Soc. Am. 86, 1511-1522 (1986).

19. Liu, J.-Y., Schmidt, H., and Kuperman, W.A., "Effect of a rough seabed on the spectral composition of deep ocean infrasonic ambient noise," J. Acoust. Soc. Am., 93, 753769 (1993).

20. Liu, J.-Y. and Krolik, J.L., "The spatial correlation of rough seabed scattering of surface-generated ambient noise,' J. Acoust. Soc. Am., 96, 2876-2886 (1994)

21. Liu, J.-Y., Wang, C., and Huang, C.F., "Coherent reflection from a rough interface over an inhomogeneous transition fluid layer," J. Comput. Acoust., 8(3), 401-414 (2000).

22. Robins, A.J., "Reflection of a plane acoustic waves from a layer of varying density," J. Acoust. Soc. Am., 87 (4), 1546-1552 (1990).
23. Robins, A.J., "Reflection of a plane wave from a fluid layer with continuously varying density and sound speed, "J. Acoust. Soc. Am., 89 (4), Pt. 1, 1686-1696 (1991).

24. Robins, A.J., "Exact solutions of the Helmholtz equation for plane propagation in a medium with variable density and sound speed," J. Acoust. Soc. Am., 93 (3), 1347-1352 (1993).

25. Schmidt, H., SAFARI: Seismo-Acoustic Fast Filed Algorithm for Range Independent Environments. User's Guide, SR 113, SACLANT ASW Research Centre, La Spezia, Italy (1987).

26. Schmidt, H., and Jensen, F.B., "A full wave solution for propagation in multilayered viscoelastic media with application to Gaussian beam reflection at a fluid-solid interfaces," J. Acoust. Soc. Am., 77, 813-825 (1985).

27. Tang, D.J., and Frisk, G., "Plane-wave reflection from a random fluid half-space,', J. Acoust. Soc. Am., 90, 27512756 (1990).

28. Uscinski, B.J., The Elements of Wave Propagation in Random Media, McGraw-Hill (1977).

29. Wolfram, S., The Mathematica Book, 3rd edi., Cambridge University Press (1996).

30. Yamamoto, T., "Geoacoustic properties of the seabed sediment critical to acoustic reverberation at 50 to 500 Hz: a preliminary data set,' J. Acoust. Soc. Am., Suppl. 185, S86 (1989).

\section{平面波自水平層化隨機流體介質粗 糙界面上之同調反射}

$$
\begin{gathered}
\text { 劉金源黄千 芬 } \\
\text { 海下技術研究所 } \\
\text { 薛 害 文 }
\end{gathered}
$$

國立中山大學海洋環境及工程學系

$$
\text { 摘 要 }
$$

本文探討平面波自具有隨機聲速擾動流體介質 粗䊁界面上之反射問題。所使用的模式, 乃結合水平 層化介質之粗糙界面散射以及隨機介質散射理論, 而 導引出適用於探討上述問題的公式。爲求得受制於兩 粗䊁邊界隨機介質中聲波方程式的解, 本文中亦推導 得符合該環境之格林函數(Green's function)。之後, 再將導得的公式, 應用在簡化的海床環境模式, 以利 探討聲波反射的問題。本研究結果顯示, 粗糙界面以 及隨機介質散射, 都將導致同調反射聲能的降低, 除 此之外, 更重要的是, 雨種機制所造成同調反射降低 的特性, 十分不同, 也因此可藉由同調反射係數的分 佈特性, 判定散射的主要機制。 